\title{
Fuzzy Cluster Analysis
}

\section{A New Method to Predict Future Cardiac Events in Patients With Positive Stress Tests}

\author{
Robert M. Peters, MD; Stanley A. Shanies, MD; John C. Peters, MD
}

\begin{abstract}
Several studies have shown that combining the change in the ST-segment with another exercise variable improves the predictive value of stress testing. However, no method has been able to combine many stress test variables with the ST-segment change simultaneously and help the clinician better predict future cardiac events. Fuzzy Cluster Analysis (FCA) was used to combine 5 stress test variables with ST-segment deviation to classify each of 232 positive outpatient stress tests as mildly, moderately, or severely abnormal. Cardiac events were recorded in these 3 patient groups up to 96 months (mean 65 months) after the stress tests. Coronary angiography was performed on 159 of these patients within 1 month of their stress tests. FCA better separated the 3 event-free survival curves than classifying the stress tests by three ST-segment $(0.5-1.5 \mathrm{~mm}, 2-2.5 \mathrm{~mm},>3 \mathrm{~mm})$ groups $(\mathrm{p}<0.05)$. At 2 years, $90 \%$ of the FCA mild group were compared with $70 \%$ for the $0.5-1.5 \mathrm{~mm}$ group $(\mathrm{p}<0.01)$. Moderate and severe tests by FCA separated patients with an intermediate from those with a poor prognosis while the $2-2.5 \mathrm{~mm}$ and $3 \mathrm{~mm}$ or more ST-segment curves did not $(\mathrm{p}<0.05)$. FCA showed overall better correlation with coronary score $(\mathrm{r}=0.71)$ than did the graded ST-segment groups $(\mathrm{r}=0.48)$. FCA predicted both mild and high-grade (triple-vessel and left main) coronary disease better than ST-segment alone. Thus FCA better predicts future cardiac events in patients with positive stress tests than the ST-segment alone. This combined with its usefulness in predicting the extent of coronary disease provides the basis of a clinical strategy for managing patients with positive stress tests. (Jpn Circ J 1998; 62: 750-754)
\end{abstract}

Key Words: Fuzzy cluster analysis; Stress test

$\mathbf{F}$ uzzy Cluster Analysis (FCA) has been shown to be better than ST depression in predicting the extent of coronary disease in patients with positive stress tests! Derived from fuzzy set theory (fuzzy logic), the method combines information from 6 stress test variables, each of which may have a range of abnormality from very mild to very severely abnormal. The resulting output classifies any positive stress test as either mildly, moderately, or severely abnormal.

Several studies have attempted to combine exercise variables to improve the prognostic value of treadmill testing?,3 We conducted this study to see if FCA is useful in predicting cardiac outcomes in outpatients with positive stress tests. Also we again looked at how well FCA predicted the extent of coronary artery disease using a larger database than in our previous study!

\section{Methods}

Study Group

Standard Bruce protocol treadmill tests from 1,955 stable outpatients were reviewed. ST-segment deviation during exercise was measured $0.06 \mathrm{sec}$ after the $\mathrm{J}$ point, and the largest absolute value for ST-segment deviation in any lead except $\mathrm{aVR}$ was recorded. Patients were selected for

(Received March 24, 1998; revised manuscript received May 18, 1998; accepted May 19, 1998)

North Shore University Hospital-Long Island Jewish Health Care System, Division of Cardiology, Long Island, New York, USA

Mailing address: Robert M Peters, MD, 1300 Union Tpke, New Hyde Park, New York 11040, USA entry into the study if they had $0.5 \mathrm{~mm}$ or more of exerciseinduced ST-segment depression or $1 \mathrm{~mm}$ or more of exercise-induced ST-segment elevation. Patients were excluded if they had had coronary angioplasty or coronary bypass surgery at any time before the stress test, if they had known significant valvular, hypertensive, or congenital heart disease, if they had significant chronic lung disease or peripheral vascular disease, if they had left bundle branch block at rest or during exercise, or if they were taking digoxin at the time of the exercise test. Patients were not excluded if they had resting ST-segment depression, Q waves, T-wave inversions, or right bundle branch block.

Two hundred and thirty-two patients (182 men and 50 women, aged 30-79 years) meeting these criteria formed the database. Typical angina was present in 130 patients, atypical symptoms were present in 74, and 28 were asymptomatic. There was a history of previous myocardial infarction in 25 patients. On the day of the stress test, 28 patients had taken nitrates, 17 had taken calcium-channel blockers, 6 had taken beta-blockers, and 41 had taken aspirin. The patients were followed up to 96 months after the stress test, with an average follow-up of 65 months (range, 8-96 months).

The time between the stress test and each patient's first cardiac event was recorded. The cardiac outcomes included in this study were: coronary bypass surgery, percutaneous translumiual coronary angioplasty (PTCA), coronary stent placement, myocardial infarction (either Q wave or nontransmural with confirmatory cardiac enzyme elevation), cardiac death, hospitalization for unstable angina, and hospitalization for congestive heart failure. Between the 
Table 1 Fuzzy Sets for Stress Test Variables

\begin{tabular}{|c|c|c|c|c|c|c|c|c|c|c|c|c|}
\hline $\begin{array}{l}\text { Degree of } \\
\text { membership }\end{array}$ & 0 & 0.1 & 0.2 & 0.3 & 0.4 & 0.5 & 0.6 & 0.7 & 0.8 & 0.9 & 1.0 & \\
\hline $\begin{array}{l}\text { Variable } \\
\text { S1 }\end{array}$ & & $\frac{1}{2} m m$ & $1 m m$ up & $1 \mathrm{~mm}$ flat & $1 m m$ down & $1-1 \frac{1}{2} m m$ & $2 \mathrm{~mm}$ flat & $\begin{array}{c}2 m m \text { down } \\
\text { or } 2 \frac{1}{2} m m\end{array}$ & $\begin{array}{l}\text { ST } \\
\text { elev. }\end{array}$ & $3 \mathrm{~mm}$ & $>3 \mathrm{~mm}$ & \\
\hline$S 2$ & 40 & $40-35$ & $35-30$ & $30-25$ & $25-20$ & $20-15$ & $15-10$ & $10-5$ & $5-(5)$ & $(5-15)$ & $>(15)$ & $(\mathrm{mmHg})$ \\
\hline S3 & 12 & $12-11$ & $11-10$ & $10-9$ & $9-8$ & $8-7$ & $7-6$ & $6-4.5$ & $4.5-3$ & $3-1$ & $<1$ & (minutes) \\
\hline$S 4$ & 100 & $100-95$ & $95-90$ & $90-85$ & $85-80$ & $80-75$ & $75-70$ & $70-65$ & $65-60$ & $60-50$ & $<50$ & (\%) \\
\hline S5 & 13 & $13-12$ & $12-11$ & $11-9$ & $9-7.5$ & $7.5-6$ & $6-4.5$ & $4.5-3$ & $3-2$ & $2-1$ & $1-0$ & (minutes) \\
\hline S6 & & $0-1$ & $1-2$ & $2-3$ & $3-4$ & $4-5$ & $5-6$ & $6-7$ & $7-9$ & $9-11$ & $>11$ & (minutes) \\
\hline
\end{tabular}

$S 1=$ degree of ST segment depression (or ST elevation greater than $1 \mathrm{~mm}$ ), $S 2=$ the difference $(\mathrm{mmHg})$ between the resting systolic blood pressure and peak exercise systolic blood pressure, $S 3=$ the total exercise (minutes) treadmill time, $S 4=$ peak exercise heart rate expressed as a \% of $100 \%$ predicted maximum for age, S5=time to onset (minutes) of angina during exercise, S6=duration (minutes) of repolarization abnormalities after termination of exercise. Numbers in parentheses represent negative values.

time of the stress test and the first cardiac event, 158 patients were taking nitrates, 100 were taking beta-blockers, 23 were taking calcium-channel blockers, 44 were taking cholesterol-lowering agents, and 112 were taking aspirin.

Of these 232 patients, 159 underwent coronary angiography within 4 weeks before or after the exercise stress test. This group included the 109 patients previously reported! For purposes of triple-vessel classification, a lesion was considered significant if it was narrowed by $50 \%$ or more by visual estimation of the angiographer. Each patient angiogram was also assigned a coronary score according to the method of Friesinger4

\section{Fuzzy Cluster Analysis}

Six stress test variables were examined for each patient: (i) ST-segment change in $\mathrm{mm}$ (S1); (ii) difference between resting systolic and peak exercise systolic blood pressure (S2); (iii) total exercise treadmill time (S3); (iv) peak exercise heart rate expressed as a percentage of $100 \%$ predicted maximal heart rate for age (S4); (v) time to onset of angina, defined as chest pain or typical anginal symptom but not including dyspnea or fatigue during exercise (S5); and (vi) duration of repolarization abnormalities (ST-segment or Twave changes or both) after termination of exercise (S6). These 6 variables were chosen because, after a general literature review, ${ }^{5-8}$ it was felt that these were most closely associated with the presence and extent of coronary artery disease. Variables such as ventricular arrhythmias, afterpotentials, and changes in R-wave height were felt to be too non-specific for this analysis. Fuzzy sets were constructed for each of these variables, with the degree of membership corresponding to the severity of the abnormality for each variable (Table 1).

Let ux $(\mathrm{Si}) \in[0,1]$ denote the membership grade in the fuzzy set characterizing the stress test of patient $X$ and defined on the set $\mathrm{S}=[\mathrm{S} 1, \mathrm{~S} 2, \mathrm{~S} 3, \mathrm{~S} 4, \mathrm{~S} 5$, S6], which indicates the severity of abnormality for the variable $\mathrm{Si}$ in the patient's stress test. To classify a patient's stress test as mildly (d1), moderately (d2), or severely (d3) abnormal, we use a clustering technique to determine to which prototypical stress test $(\mathrm{d} 1, \mathrm{~d} 2, \mathrm{~d} 3)$ the patient's stress test is most similar. The prototypes of mildly, moderately, and severely abnormal stress tests are based on published reviews ${ }^{5-8}$ for the range of each variable and are described by the matrixes:

$$
\begin{aligned}
& \text { S1 S2 S3 S4 S5 S6 } \\
& \mathrm{d} 1=\begin{array}{l}
\text { lower } \\
\text { upper }
\end{array}\left[\begin{array}{llllll}
.1 & 0 & 0 & 0 & 0 & .1
\end{array}\right] \\
& \text { S1 S2 S3 S4 S5 S6 }
\end{aligned}
$$

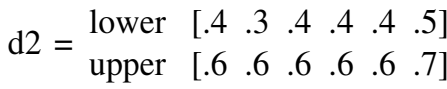

$$
\begin{aligned}
& \text { S1 S2 S3 S4 S5 S6 } \\
& \mathrm{d} 3=\begin{array}{l}
\text { lower } \\
\text { upper }
\end{array}\left[\begin{array}{cccccc}
.7 & .7 & .7 & .7 & .7 & .8
\end{array}\right]
\end{aligned}
$$

We further define a fuzzy relation $\mathrm{W}$ on the set of variables and prototypes that specify the importance or pertinence of each variable $\mathrm{Si}$ in these prototypes. The relation $\mathrm{W}$ of these weights of relevance is also based on general published reviews- and given by:

$$
\mathrm{W}=\begin{array}{cccc} 
& \mathrm{d} 1 & \mathrm{~d} 2 & \mathrm{~d} 3 \\
\mathrm{~S} 1 & .8 & .9 & .7 \\
\mathrm{~S} 2 & .5 & .5 & .2 \\
\mathrm{~S} 3 & .5 & .6 & .4 \\
\mathrm{~S} 4 & .4 & .5 & .4 \\
\mathrm{~S} 5 & .6 & .7 & .4 \\
\mathrm{~S} 6 & .5 & .6 & .4
\end{array}
$$

Let $u_{w}\left(S_{i}, d_{j}\right)$ denote the weight of each variable for each prototype $\mathrm{d}_{\mathrm{j}}$. To determine which prototype (mild $\mathrm{d} 1$, moderate $d 2$, severe $d 3$ ) is most similar to the patient's stress test, we compute a similarity measure between the patient's stress test variables and those typical of each prototype $\left(d_{j}\right)$. This similarity measure, based on the Minkowski distance using a modified Euclidean metric $(\mathrm{p}=2)$, is given by the formula:

$$
\begin{aligned}
& D_{p}\left(d_{j}, \mathrm{X}\right)=\left[\sum_{E_{A j}}\left|u_{w}\left(S_{i}, d_{j}\right)\left(u_{d j 1}\left[S_{i}\right]-u_{x}\left[S_{i}\right]\right)\right|^{P}+\right. \\
& \left.\sum_{E_{B j}}\left|u_{w}\left(S_{i}, d_{j}\right)\left(u_{d j u}\left[S_{i}\right]-u_{x}\left[S_{i}\right]\right)\right|^{P}\right]^{1 / p} \\
& \text { where } \quad A_{j}=\left[i \mid u_{x}\left(S_{i}\right)<u_{d j 1}\left(S_{i}\right), 1 \leq i \leq m\right] \\
& B_{j}=\left[i \mid u_{x}\left(S_{i}\right)>u_{d j u}\left(S_{i}\right), 1 \leq i \leq m\right]
\end{aligned}
$$

Where $\mathrm{m}$ equals the total number of variables. The similarity measure that yields the minimal value is the prototype stress test that most closely resembles the patient's stress test. A similar fuzzy cluster method was used by Esogbue and Elder in examining the similarity between a patient's symptoms and the prototypical symptom patterns seen in various diseases?,10 


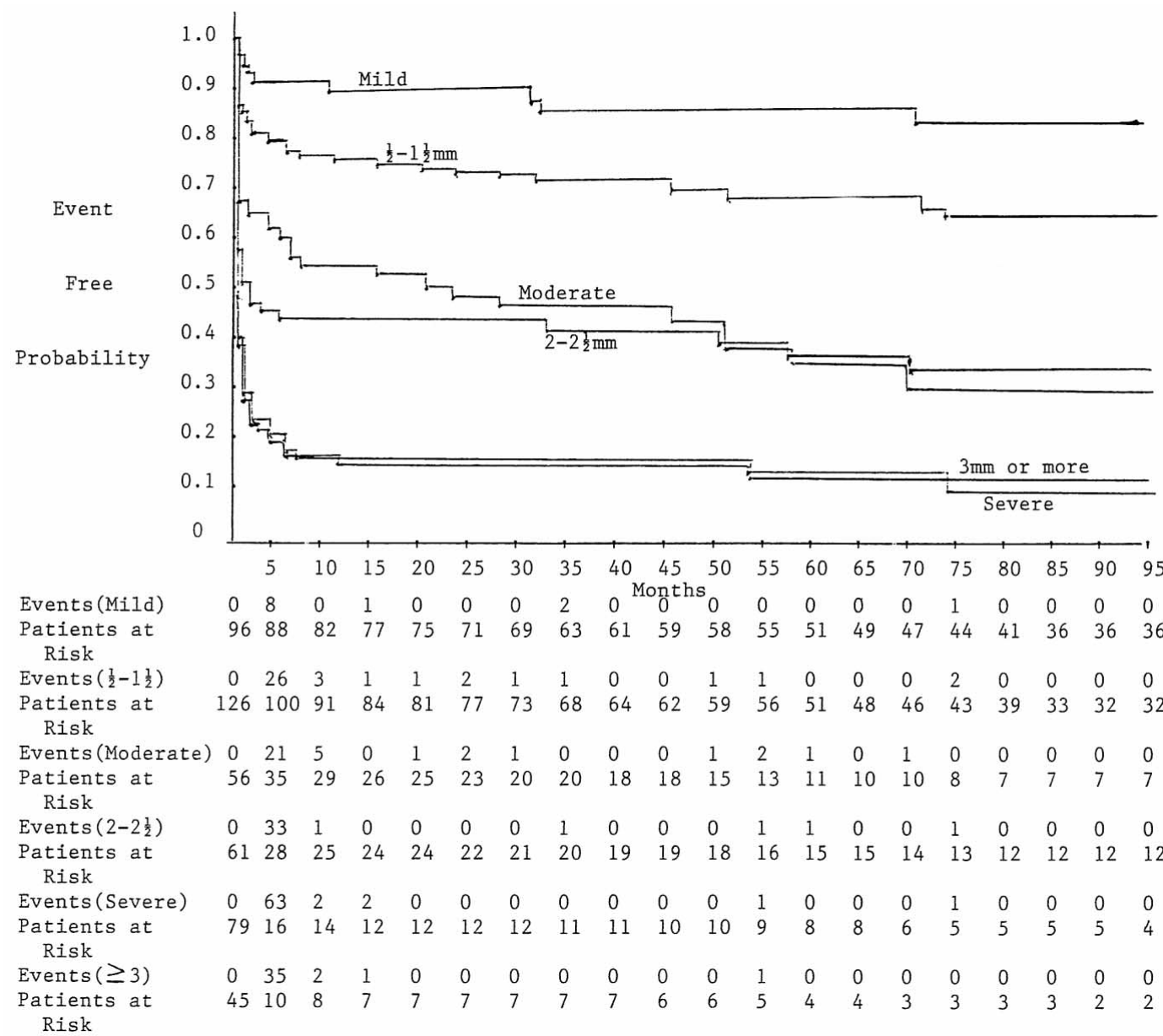

Fig 1. Event-free survival curves for the fuzzy cluster analysis groups (mild, moderate, severe) and the ST-segment depression groups.

\section{Sample Calculation}

A patient underwent an exercise stress test with the following results: ST-segment, $1.5 \mathrm{~mm}$ horizontal depression $(\mathrm{S} 1=0.5)$; systolic blood pressure change, $20 \mathrm{~mm}$ decrease $(\mathrm{S} 2=1)$; total exercise time, $5 \mathrm{~min}(\mathrm{~S} 3=0.7)$; peak heart rate, $85 \%$ predicted maximum for age $(\mathrm{S} 4=0.4)$; time to angina, $2 \mathrm{~min} 50 \mathrm{sec}$ ( $\mathrm{S} 5=0.8$ ); and duration of repolarization abnormalities, $9 \mathrm{~min}(\mathrm{~S} 6=0.9)$.

$$
\begin{aligned}
\mathrm{D} 2(\mathrm{~d} 1, \mathrm{x}) & =\left[\{(0.8)(0.3-0.5)\}^{2}+\{(0.5)(0.2-1)\}^{2}\right. \\
& +\{(0.5)(0.3-0.7)\}^{2}+\{(0.4)(0.3-0.4)\}^{2} \\
& \left.+\{(0.6)(0.3-0.8)\}^{2}+\{(0.5)(0.4-0.9)\}^{2}\right]^{1 / 2} \\
& =0.616 \\
\mathrm{D} 2(\mathrm{~d} 2, \mathrm{x})= & {\left[\{(0.5)(0.6-1)\}^{2}+\{(0.6)(0.6-0.7)\}^{2}\right.} \\
& \left.+\{(0.7)(0.6-0.8)\}^{2}+\{(0.6)(0.7-0.9)\}^{2}\right]^{1 / 2} \\
& =0.279 \\
\mathrm{D} 2(\mathrm{~d} 3, \mathrm{x})= & {\left[\{(0.7)(0.7-0.5)\}^{2}+\{(0.4)(0.7-0.4)\}^{2}\right]^{1 / 2} } \\
& =0.184
\end{aligned}
$$

Therefore, this patient's stress test is most similar to the prototype $\mathrm{d} 3$ (severe) because this similarity measure yields the minimal value. The patient would be classified as having a severely abnormal stress test.

\section{Statistical Analysis}

The probability of event-free survival was plotted over time with Kaplan-Meier life tables. Differences between groups in event-free survival were tested with log-rank statistics. All correlations were determined by calculation of Pearson's coefficient of correlation. Differences in continuous variables were compared using Student's t test, whereas differences of categoric variables were compared using the Chi-square test. All tests were two-tailed, and a $\mathrm{p}$ value of $<0.05$ was considered statistically significant.

\section{Results}

\section{Cardiac Outcomes}

A total of 116 cardiac events took place for the entire 232 patient study group: 62 coronary bypass operations, 28 coronary angioplasty procedures, 12 myocardial infarctions, 7 admissions for unstable angina, 3 coronary stent placements, 2 admissions for congestive heart failure, and 2 cardiac deaths. Classifying the patient stress tests into 3 groups (mild, moderate, severe) by FCA better separated the 3 event-free survival curves (Fig 1 ) than classifying the stress tests by $3 \mathrm{ST}$-segment groups $(0.5-1.5 \mathrm{~mm}, 2-2.5$ $\mathrm{mm}$, and $>3 \mathrm{~mm}$ ). For FCA, overall log-rank statistic $(\mathrm{c} 2=64.5)$ was much greater than for the ST-segment classification $(\mathrm{c} 2=29.6)$.

The 96 patients classified as mild by FCA had a benign course with few cardiac events: 8 PTCA, 2 coronary bypass operations (one occurring 72 months after the stress test), and 2 non-fatal myocardial infarctions (one occurring 33 months after the stress test). At 2 years from the time of the 

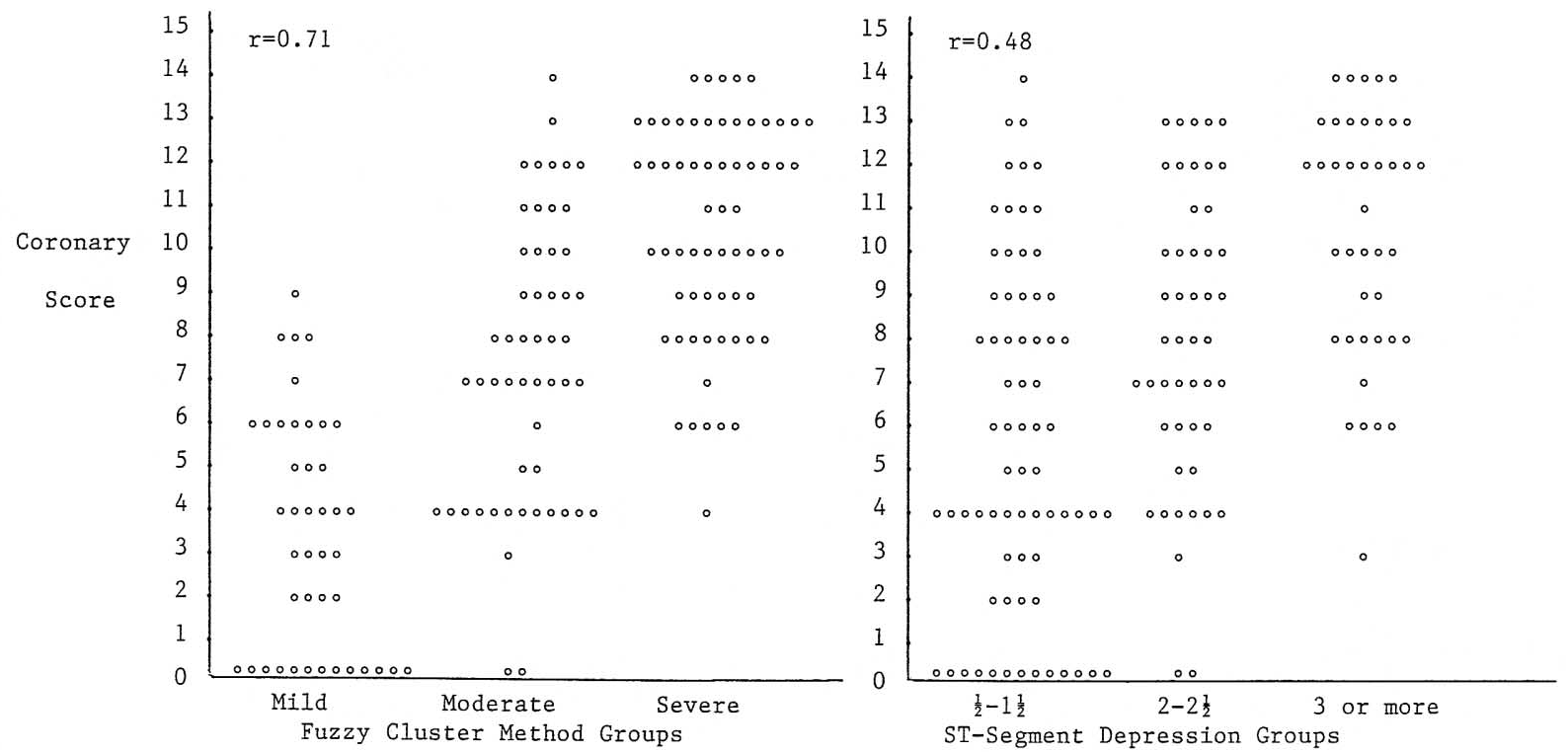

Fig 2. Coronary artery score plotted against fuzzy cluster method groups and graded ST-segment depression groups.

stress test, $90 \%$ of the mild FCA group were event-free compared to $70 \%$ for the $0.5-1.5 \mathrm{~mm}$ group $(\mathrm{p}<0.01)$. The 2 cardiac deaths in the study occurred in the $0.5-1.5 \mathrm{~mm}$ group: these 2 tests were classified as severely abnormal by FCA.

The difference between the FCA moderate and severe curves was statistically significant $(p<0.05)$, thus separating patients with an intermediate prognosis from those with a poor prognosis. The difference between the $2-2.5 \mathrm{~mm}$ and $3 \mathrm{~mm}$ or more ST-segment curves was not statistically significant, so the separation between intermediate and poor prognostic groups could not be made on the basis of the ST-segment. Thus FCA appears better than ST-segment classification in separating patients into good, intermediate, and poor prognostic categories for cardiac events.

\section{Extent of Coronary Disease}

Fuzzy Cluster Analysis showed overall better correlation (Fig 2) with coronary score ( $\mathrm{r}=0.71)$ than did graded STsegment groups $(\mathrm{r}=0.48)$. A test classified as mild by FCA essentially excluded high-grade coronary disease. No patient classified as mild had a coronary score above 9 , only $12 \%$ had a coronary score above 6 , and none had triple-vessel or left main disease. This is in contrast to the $0.5-1.5 \mathrm{~mm}$ ST group in which $27 \%$ had a coronary score of 9 or more, and $17 \%$ had triple-vessel or left main disease.

Tests classified as severe by FCA were associated with high-grade coronary disease. For patients with triple vessel disease, $76 \%$ had stress tests classified as severely abnormal by FCA, whereas only $43 \%$ of tests in patients with triple vessel disease showed $3 \mathrm{~mm}$ or more ST depression. Also for triple vessel disease, $24 \%$ of the stress tests showed 1/2-1 1/2 mm ST depression, but none were classified as mild by FCA. For patients with left main disease, $84 \%$ of their tests were classified as severe, whereas only $58 \%$ of patients with left main disease had tests showing 3 $\mathrm{mm}$ or more ST depression (Table2). Interestingly, in the 1/2-1 1/2 mm ST group, the 2 patients with left main disease had stress tests classified as severely abnormal by FCA. Thus whatever the degree of ST-segment depression,
Table 2 Distribution of Coronary Artery Disease in the Fuzzy Cluster Analysis and ST-Segment Depression Groups

\begin{tabular}{lccccc}
\hline \hline & \multicolumn{5}{c}{ No coronary arteries narrowed $>50 \%$} \\
\cline { 2 - 6 } & $\begin{array}{c}\text { Normal } \\
(n=15)\end{array}$ & $\begin{array}{c}1 \\
(n=41)\end{array}$ & $\begin{array}{c}2 \\
(n=42)\end{array}$ & $\begin{array}{c}3 \\
(n=42)\end{array}$ & $\begin{array}{c}\text { Left main } \\
(n=19)\end{array}$ \\
\hline Mild (42) & 13 & 21 & 8 & 0 & 0 \\
$\frac{1}{2}-1 \frac{1}{2}$ mm (70) & 13 & 26 & 19 & 10 & 2 \\
Moderate (53) & 2 & 18 & 20 & 10 & 3 \\
$2-2 \frac{1}{2}$ mm (48) & 2 & 13 & 13 & 14 & 6 \\
Severe (64) & 0 & 2 & 14 & 32 & 16 \\
$3 m m^{*}(41)$ & 0 & 2 & 10 & 18 & 11 \\
\hline
\end{tabular}

*Includes two patients with ST-segment elevation.

tests classified as severe by FCA are strongly associated with high-grade coronary disease.

Patients classified as moderately abnormal by FCA had a wide range of coronary disease (from single vessel to left main) and a similar wide range of coronary scores (from 3 to 14). Thus, as in the $2-2.5 \mathrm{~mm}$ ST group, tests classified as moderate were not as helpful in predicting the extent of coronary disease.

\section{Discussion}

Several stress test studies have shown that various ways of combining the ST-segment response with data from other exercise variables adds more prognostic information than looking at the ST-segment alone , $^{3}$ However, there is no standard way of combining exercise variables with the ST-segment, and studies regarding the importance of different stress test variables in predicting the extent of coronary disease have yielded various results! Thus only general guidelines have emerged to help clinicians interpret positive stress tests and predict cardiac events and extent of coronary disease.

This may be due in part to the fact that these studies mainly use Bayesian probability methods or discriminant function analysis, both of which rely on sharp cutoff points 
between abnormality and normality for a given variable, thus ignoring both the gradation of abnormality present in each of these variables, and information from variables in the mildly abnormal or normal range in the analysis!1,12 This may not be appropriate for situations where there is a gradation of abnormality from mildly to severely abnormal. All commonly used stress test variables, the degree of underlying coronary disease, and the interpretation of the stress test itself have such a continuum from very mild to very severely abnormal.

Fuzzy set theory (fuzzy logic) has been developed as an alternative method of handling data having a graded degree of abnormality! ${ }^{13}$ The theory retains a strong linguistic connection with commonly used descriptive terms such as moderately abnormal or very severe ${ }^{14}$ The primary advantage of FCA, a method derived from fuzzy set theory, is that it incorporates into the analysis a graded degree of abnormality for each stress test variable, adds a weight to each variable, combines many variables at the same time, and then generates an easily understood interpretation of the entire stress test 15 Thus each variable, even if in the normal range, adds some useful information in determining how abnormal each stress test is. In these respects, the method resembles the subjective reasoning process that physicians go through as they look at each exercise variable when interpreting a positive stress test. A neuro-fuzzy algorithm was used by Sztandera et al to aid in the interpretation of stress Thallium scintigraphy! 16 However, the method used in the present study is the first to combine exercise stress test variables with FCA.

In this study, FCA was more useful in predicting future cardiac events than the ST-segment alone. FCA also showed a better relationship to the extent of coronary disease, and better predicted both mild and high-grade disease than the ST-segment alone. Based on these results, FCA could be used as a basis of a clinical strategy.

Most stable outpatients could be initially evaluated with a standard treadmill exercise test rather than a more expensive nuclear stress test or stress echocardiography. Patients with mildly abnormal tests by FCA could generally be treated medically, while those with severely abnormal tests by FCA could be strongly considered for angiography. For those with moderately abnormal tests (associated with an intermediate prognosis and a wide range of coronary disease), the clinician may then choose nuclear or stress echocardiography to better delineate which patients in this group may have high-grade disease and hence a poor prognosis. Although not specifically studied in this paper, this strategy could result in considerable cost savings. In addition, FCA can be done quickly, despite the complexity of the formulas. Once familiar with the calculations, the analysis can be done in less than 3 min using a handheld calculator, and in much less time using computer software.

\section{Acknowledgments}

The authors wish to thank the following physicians who contributed to this study: Daniel Appelbaum, James LaRosa, David Mykoff, Peter Orlic, Gerald Schattner, Joseph Schattner, Kevin Tack and Stephen Tranchina.

\section{References}

1. Peters R, Shanies S, Peters C: Fuzzy cluster analysis of positive stress tests, a method of combining exercise test variables to predict extent of coronary artery disease. Am J Cardiol 1995; 76: 648-651

2. Mark D, Shaw L, Harrell F, et al: Prognostic value of a treadmill exercise score in outpatients with suspected coronary artery disease. N Engl J Med 1991; 325: 849-853

3. Mark D, Hlatky M, Harrell F, Lee K, Califf R, Pryor D: Exercise treadmill score for predicting prognosis in coronary artery disease. Ann Intern Med 1987; 106: 793-799

4. Moise A, Clement B, Saltiel J: Clinical and angiographic correlates and prognostic significance of the coronary extent score. Am J Cardiol 1988; 61: 1255-1259

5. Froelicher V, Myers J, Follansbee W, Labovitz A: Exercise and the heart. St Louis: Mosby, 1993: 136-142

6. Dubach P, Froelicher V, Klein J, Oakes D, Grover-McKay M: Exercise induced hypotension in a male population. Circulation 1988; 78: $1380-1387$

7. Cole J, Ellestad M: Significance of chest pain during treadmill exercise: correlation with coronary events. Am J Cardiol 1978; 41: 227 235

8. Ellestad M, Wan M: Predictive implications of stress testing: followup of 2700 subjects after maximum treadmill stress testing. Circulation 1975; 51: $363-375$

9. Esogbue A, Elder R: Measurement and validation of a fuzzy mathematical model for medical diagnosis. Fuzzy Sets Syst 1983; 10: $223-$ 242

10. Esogbue A, Elder R: Fuzzy sets and the modeling of physician decision processes, part II, fuzzy diagnosis decision models. Fuzzy Sets Syst 1980; 3: $1-9$

11. Rifkin R, Hood W: Bayesian analysis of electrocardiographic exercise testing. N Engl J Med 1977; 297: 681-685

12. Young M, Fried L, Eisenberg J, Williams S: The single-cutoff trap: implications for Bayesian analysis of stress electrocardiograms. Med Decis Making 1989; 9: 176-180

13. Zimmerman H: Fuzzy set theory and its application. Boston: Kluwer Academic Publishers, 1991: 178-190

14. McNeill D, Freilberger P: Fuzzy logic. New York: Simon \& Schuster, 1993: $23-88$

15. Klir G, Folger T: Fuzzy sets, uncertainty, and information. Englewood Cliffs: Prentice-Hall, 1988: 252-254

16. Sztandera L, Goodenday L, Cios K: A neuro-fuzzy algorithm for diagnosis of coronary artery stenosis. Comput Biol Med 1996; 26: $97-111$ 